\title{
Le dictionnaire de chimie en lingála pour les élèves de Kinshasa
}

\author{
Bienvenu Sene Mongaba, Département de Langues et \\ Cultures africaines, Université de Gand, Belgique \\ (senemongaba@yahoo.fr)
}

Résumé: Pour réaliser un dictionnaire spécialisé dans une langue bien documentée, le lexicographe dépouille les documents disponibles, il en extrait des termes candidats et, éventuellement, leurs définitions et des exemples pertinents. Cette approche n'est pas adaptée pour des langues dont les documents correspondant à la spécialité sont rares ou absents. Tel est le cas du lingála, langue parlée dans la ville de Kinshasa, dans le domaine de la chimie. Outre le travail de dépouillement du corpus, des travaux de création terminologique doivent donc être réalisés, afin de permettre à ces dictionnaires de satisfaire les attentes des utilisateurs. Pour cela, le lexicographe adopte l'une des deux méthodologies suivantes.

La première méthodologie, fréquemment utilisée, procède par la traduction. Elle consiste à utiliser un corpus dans une langue source, en extraire des lemmes, des définitions et des exemples et, enfin, classifier ces données, toujours dans la langue source. Le document ainsi obtenu est ensuite traduit dans la langue cible.

La deuxième méthodologie consiste à définir des concepts et à produire les termes les désignant directement dans la langue source, sans pour autant toujours disposer d'un corpus correspondant dans cette langue. Cette approche nécessite donc que le lexicographe soit lui-même expert dans le domaine en question ou qu'il travaille avec un expert, qui va faire ce travail de production du savoir dans la langue cible. Ensuite, le lexicographe soumet ses résultats à la validation de locuteurs experts. Dans ce travail, nous avons adopté et nous décrivons cette deuxième approche.

En effet, cet article décrit la démarche que nous avons suivie pour la réalisation d'un dictionnaire de chimie bilingue lingála-français à l'intention des enseignants et des élèves de la troisième année secondaire des écoles de Kinshasa.

Mots-clés: TERMINOLOGIE, LEXICOGRAPHIE DES TERMES DE SPÉCIALITÉ, LINGÁLA, CONGO, KINSHASA, DICTIONNAIRE PÉDAGOGIQUE, DICTIONNAIRE DIDACTIQUE, DICTIONNAIRE BILINGUE

Abstract: Dictionary of Chemistry in Lingála for Kinshasa Students. In welldocumented languages, the lexicographer aiming to make a specialized dictionary will process an available corpus to extract data (candidate terms, definitions and examples). This approach is not suitable for poorly documented languages, for which texts in any given specialized domain may be few and far between. This is the case with Lingála, spoken in the city of Kinshasa, in the field of chemistry. Besides processing the available corpora, the lexicographer needs to coin terms for concepts and to create definitions and examples in order to produce dictionaries meeting users' expectations. For this purpose, one of the two following methodologies can be adopted.

Lexikos 23 (AFRILEX-reeks/series 23: 2013): 548-564 
The first, and more commonly employed methodology adopts the translation approach. A corpus which is available in the source language is processed to extract lemmas, definitions and examples and eventually classify data in the source language. The document obtained in this way is then translated into the target language.

According to the second methodology, in the absence of well-documented specialized corpora in the target language, concepts are defined and coined directly in the target language. Examples are also produced directly in the target language. This approach calls for the lexicographer to be an expert in the given field or for him/her to work with an expert who can produce specialized knowledge in the target language. Then the lexicographer will submit his/her findings to other native speakers, who are also experts in that given field for validation. This is the methodology we have adopted and we are to describe in this paper.

In fact, this article describes the approach we have followed in order to make a LingálaFrench bilingual dictionary of chemistry for students and teachers of the third year of secondary school in Kinshasa.

Keywords: TERMINOLOGY, LSP LEXICOGRAPHY, LINGÁLA, CONGO, KINSHASA, CHEMISTRY, PEDAGOGIC TOOL, PEDAGOGICAL DICTIONARY, DIDACTIC DICTIONARY, BILINGUAL DICTIONARY

\section{Introduction}

En République Démocratique du Congo, le français est utilisé comme langue d'enseignement et il jouit d'un privilège du point de vue représentationnel de la part de l'institution scolaire. Néanmoins, les élèves des écoles de la RD Congo, en particulier ceux des écoles de la ville de Kinshasa, maîtrisent de moins en moins le français, comme le montrent la plupart des études (Manduku Sasa 2004; Nyembwe et Koni 2004; Yawidi 2009; Nyembwe et Matabishi 2012; Kilosho 2013). Les enquêtes sociolinguistiques réalisées entre 2008 et 2012 (Sene Mongaba 2013b) ont révélé que, dans la ville de Kinshasa, capitale de la $\mathrm{RD}$ Congo, les enseignants sont souvent obligés de recourir au lingála pour réexpliquer les leçons pourtant données en français. En revanche, ils n'ont d'autre choix que d'employer un discours non spécialisé en lingála, puisque cette langue n'est pas enseignée à l'école et que les supports didactiques y font cruellement défaut. Les enseignants et les élèves utilisent donc la langue parlée dans la rue et dans le cercle familial. Ceci constitue un handicap dans le processus de transmission du savoir en milieu scolaire, qui exige un langage de spécialité adapté aux savoirs enseignés/appris. L'une des solutions consiste à confectionner des dictionnaires de spécialités qui pourront aider les enseignants et les élèves à construire un discours spécialisé cohérent.

Cet article décrit la démarche suivie pour la réalisation d'un dictionnaire de chimie bilingue lingála-français à l'intention des élèves et enseignants de la troisième année secondaire des écoles de Kinshasa.

Cet article comprend sept sections. Après cette introduction, la deuxième section traite du cadre théorique et méthodologique. La troisième section décrit 
l'utilisateur-cible, tandis que la quatrième section expose les objectifs du dictionnaire. La cinquième section montre la démarche suivie pour sélectionner les lemmes et produire des définitions. La présentation de la structure du dictionnaire intervient à la sixième section qui précède la conclusion.

\section{Le cadre théorique et méthodologique: L'extraction des données ter- minologiques}

La terminologie, conçue comme une discipline scientifique prend en compte, comme le soutient Depecker (2009: 97), "les unités linguistiques dans les langues, les concepts par lesquels le réel est représenté dans la pensée, les objets et les représentations". La méthode adoptée dans un travail de terminologie dépend des objectifs poursuivis et des ressources disponibles. Chaque langue a une organisation bien définie à laquelle obéissent les unités linguistiques qui constituent les matériaux dont le terminologue a besoin pour organiser la terminologie dans un domaine bien précis. À côté de cette connaissance linguistique, les concepts, ainsi que les objets à nommer font partie d'un domaine, qui n'est pas la linguistique mais un domaine d'activités, par exemple la chimie, qui a aussi sa nomenclature et son système notionnel propre. La connaissance de cette organisation est également nécessaire dans un travail de terminologie. Enfin, les personnes, à qui est destinée cette terminologie, ont leurs représentations, aussi bien linguistiques que scientifiques ou technologiques, qu'il ne faut pas non plus ignorer.

Deux étapes préalables à la confection des dictionnaires sont importantes en lexicographie. Il s'agit de décrire les caractéristiques de l'utilisateur-cible et de déterminer les objectifs du dictionnaire que l'on veut réaliser. Ces deux étapes dictent les décisions que le lexicographe prendra, par la suite, pour la sélection des lemmes, des définitions, des exemples, du niveau de langue, ainsi que le choix des champs que va comporter chaque article (Prinsloo et al. 2000).

Aussi bien pour la terminologie que pour la lexicographie, l'extraction des termes/lemmes candidats marque le début du travail productif proprement dit. Dans plusieurs langues bien documentées, comme par exemple le français ou l'anglais, les terminologues et les lexicographes passent par le dépouillement des textes disponibles pour en extraire les termes candidats et éventuellement leurs définitions. Cette approche n'est pas adaptée à plusieurs langues africaines - tel est le cas du lingála - à cause de la faible quantité de documentation disponible. Cette faible production de textes ne permet pas un travail terminologique et lexicographique sur base de corpus écrit. En plus donc du travail de dépouillement du corpus, des travaux de création terminologique et de production de contenu doivent être réalisés, afin de permettre à ces dictionnaires de satisfaire les attentes des enseignants et des élèves. Pour cela, les lexicographes des langues africaines adoptent l'une des deux méthodologies suivantes.

La première méthodologie, fréquemment utilisée, consiste à utiliser un 
corpus dans une langue source (comme l'anglais ou le français), en extraire des lemmes, des définitions et des exemples et pour enfin classifier ces données dans cette langue source. Le document classifié est ensuite traduit dans la langue cible (Matumele et al. 1995; Diki-Kidiri 2008: 113; Taljard et Nchabeleng 2011). Telle est l'approche que nous appelons traductionnelle.

Nous avons en revanche adopté la deuxième méthodologie, que nous qualifions d'approche terminologique. En effet, nous avons rédigé, en lingála, un manuel de chimie conforme au programme de la troisième année secondaire. Ainsi, nous avons défini les concepts et produit des exemples de contextualisation directement dans la langue cible. Ce travail de définition de concepts et de production d'exemples a ensuite permis la création de termes de spécialité qui se base sur les mécanismes de création de termes en lingála, notamment, la dérivation, la composition et l'emprunt (Sene Mongaba 2012). Cette approche a été possible grâce à notre parcours de chimiste d'une part et de linguiste d'autre part. Bien sûr, si le lexicographe n'est pas en même temps expert du domaine de création des termes, il va se prévaloir de la collaboration d'un expert, mais il sera malgré tout crucial, dans l'approche que nous préconisons, que l'expert produise effectivement du savoir dans la langue cible. Dans cette approche, la connaissance approfondie des mécanismes linguistiques de la langue cible permet la systématisation des méthodes de créations des termes, ainsi que le renforcement des capacités de cette langue.

Il y a lieu de faire une distinction entre cette approche "terminologique" et une approche de "lexicologie spécialisée". En effet, nous adoptons la définition de la lexicologie spécialisée comme étant "la branche de la lexicologie qui examine les unités lexicales des discours spécialisés en général au niveau formel et abstrait afin de dégager des méthodes de repérage, d'analyse et de description des unités lexicales qui lui sont spécifiques" (Valente 2002: 68-71). Nous n'analysons pas les unités lexicales de textes produits en lingála mais nous produisons, en lingála, un texte spécialisé. C'est à partir de ce texte spécialisé que nous extrayons la définition du concept et sur base de termes principaux contenus dans cette définition que nous déduisons une dénomination.

Cette approche n'est pas non plus seulement terminographique, dans la mesure où elle ne se limite pas à extraire et à ranger des termes et leurs définitions, mais, elle aborde aussi l'aspect théorique "en orientant l'usager vers une solution terminologique acceptable linguistiquement et qui répondent pleinement à ses attentes de locuteurs" (Auger 2001: 220-221). La théorie adoptée dans cette approche est liée à la réalisation d'un dictionnaire bilingue à l'intention des élèves dont la langue première est une langue faiblement documentée alors que leur langue d'enseignement est une langue documentée.

Le lecteur gardera à l'esprit que ces deux approches - traductionnelle et terminologique - sont complémentaires, dans la mesure où l'approche traductionnelle permet une production quantitative de termes de spécialité dans un temps relativement court, alors que l'approche terminologique permet d'éprouver l'insertion des termes créés dans la syntaxe et la sémantique de la langue 
cible et éventuellement y apporter des modifications. Enfin, le contexte culturel et socio-économique de la communauté linguistique cible y est aussi automatiquement intégré.

\section{La caractérisation de l'utilisateur cible}

Le dictionnaire de chimie visé dans cette démarche est destiné aux élèves et aux enseignants de la troisième année secondaire scientifique de la ville de Kinshasa.

Du point de vue du contenu, au Congo, le cours de chimie commence en troisième année secondaire, c'est-à-dire à la neuvième année d'école. Le Ministère de l'enseignement secondaire a publié un programme national de chimie que toutes les écoles sont tenues de respecter (Ministère EPSP 2009). À cet effet, plusieurs livres de chimie, écrits en français et conformes à ce programme, sont édités par des entreprises privées. D'une manière globale, le programme de chimie de troisième année secondaire, à raison de trois heures par semaine, prévoit les matières suivantes:

- les notions fondamentales (définition de chimie, définition de la matière, la transformation chimique et physique),

- l'étude de l'atome et de la molécule,

- la réaction chimique,

- la description de certains atomes, molécules ou mélanges tels que le chlore, l'oxygène, l'eau, l'air, le feu, etc.

Du point de vue sociolinguistique, il existe plusieurs variétés de lingála. Dans ce travail, nous nous intéresserons à la variété dite lingála ya Kinshasa qui correspond à la variété utilisée dans les villes de Kinshasa et Brazzaville. Cette variété a quatre principaux registres suivants:

- Le lingála ya sóló: il s'agit du registre élaboré. Il est utilisé dans les textes littéraires, les manuels de métiers, les revues de sensibilisation, ainsi que dans les versions récentes de la Bible et du Coran publiées après l'année 2000.

- Le lingála facile: il s'agit du lingála parlé dans ces deux villes. Il est désigné sous ce vocable à cause de son taux élevé de mots français incorporés dans le discours des locuteurs.

- Le lingála ya bayanké (indoubill): il s'agit du registre argotique.

- Le langíla: il s'agit d'un registre encrypté du lingála ya bayanké.

Le lecteur peut se référer à l'article de Sene Mongaba (2012) en ce qui concerne les détails sur les variétés et les registres du lingála. 
Nos enquêtes ont montré que, dans la ville de Kinshasa, le taux de mots français dans le lingála est d'environ $25 \%$ pour un locuteur faiblement instruit. Ce pourcentage peut s'élever jusqu'à $50 \%$ ou plus pour un locuteur instruit (Sene Mongaba 2013a: 15-135). Les élèves de la troisième année secondaire parlent couramment le lingála facile au taux moyen de $25 \%$. Ils sont aussi relativement bien exposés aux textes écrits dans ce registre par l'internet (Facebook, Youtube, forum des sites congolais, ...), des SMS, de la radio et de la télévision. Il s'agit, souvent, de textes traitant les affaires de la vie courante (musique, théâtre, politique, religion, ...). Puisque, comme nous le rappelions plus haut, le lingála n'est pas enseigné à l'école, les élèves n'ont donc qu'un très faible accès aux textes élaborés (scolaires, littéraires, ...), aussi bien en lingála facile qu'en lingála ya sóló.

Il est utile de souligner à nouveau que le fait que les locuteurs du lingála facile utilisent un taux élevé de mots français ne signifie pas que ces locuteurs ont une bonne maîtrise de la langue française. En effet, ces mots sont utilisés, en grande partie, dans la syntaxe du lingála et souvent comme des emprunts et non comme de l'alternance codique.

Les élèves de la troisième année secondaire ont en moyenne 15 ans. Hormis les élèves des écoles élitistes et de quelques écoles des milieux aisés, les élèves des écoles secondaires de la ville de Kinshasa ont, en général, une très faible maîtrise du français (lire, écrire, parler, écouter, comprendre et reformuler). Telle est la raison pour laquelle les 58 professeurs de chimie interviewés dans le cadre d'une autre enquête sur la diglossie français-lingála (Sene Mongaba 2013b) sont convaincus qu'un dictionnaire bilingue lingála-français est nécessaire pour pouvoir réexpliquer les termes de chimie qui sont utilisés en français en classe.

Les élèves de la troisième année secondaire sont dans leur première année de chimie. Les définitions proposées dans le dictionnaire sont donc destinées à des novices en chimie, tout en gardant à l'esprit qu'il ne s'agit pas d'un document de vulgarisation. Ceci signifie que les définitions ainsi que les exemples devront être faits dans un discours de spécialité (chimie) sans toutefois rentrer dans des détails qui sortent du cadre de programme de chimie de la troisième année secondaire scientifique.

En ce qui concerne les enseignants, les métadonnées de l'enquête précitée auprès des 58 enseignants de chimie pour la validation des créations terminologique en lingála ont permis de caractériser le type d'enseignants de chimie que l'on peut rencontrer dans une classe de troisième année secondaire dans les écoles de Kinshasa. Les enseignants de la troisième année secondaire sont diversement qualifiés par rapport à l'enseignement de la chimie. Selon les règlements en RD Congo, les enseignants qualifiés sont ceux qui disposent d'un diplôme de trois ans (premier cycle universitaire) ou d'un diplôme de cinq ans (premier et deuxième cycle universitaire) en pédagogie option chimie-biologie. 30 enseignants (52\%) remplissaient cette condition, 20 enseignants $(34 \%)$ ont fait des études de chimie ou de biologie dans les filières des facultés des 
sciences sans formation en pédagogie et 8 enseignants (14\%) sont des personnes faiblement formées, ne disposant que de leur diplôme d'enseignement secondaire ou ayant fait, au plus, une ou deux années d'université.

Nous avons évalué la compétence linguistique des enseignants en lingála facile sur base de nombre d'années vécu à Kinshasa ainsi que sur leur capacité à répondre dans cette langue au cours des interviews. 25 enseignants sont nés à Kinshasa (43\%), 23 vivent à Kinshasa depuis plus de 10 ans (40\%), 6 vivent à Kinshasa entre 5 et 10 ans (10\%) et enfin 4 habitent à Kinshasa depuis moins de 5 ans (7\%). 56 enseignants ont répondu à nos questions en lingála facile, tandis que seulement 2 d'entre eux ont affirmé ne pas pouvoir le faire (3\%). Cette enquête nous autorise à dire qu'en général ces enseignants ont les compétences linguistiques en tant que locuteurs de lingála mais ne disposent pas, de façon consciente, de la connaissance du fonctionnement des mécanismes de dérivation et de composition du lingála.

\section{Les objectifs du dictionnaire}

L'objectif poursuivi est celui de mettre à la disposition des élèves et des enseignants de la troisième année secondaire scientifique de Kinshasa un dictionnaire lingála-français qui servira de support pour la compréhension du cours de chimie. Deux situations possibles peuvent résumer les besoins des élèves et des enseignants.

Situation 1. Les élèves ont besoin de comprendre la signification d'une notion donnée au cours. Il s'agit donc de trouver l'explication en lingála d'un mot français. Le mot peut être un terme de chimie ou un mot de la langue générale. L'entrée de l'article sera donc en français mais devra comporter la définition et des exemples en lingála. Le terme équivalent en lingála favorise aussi la compréhension du concept et du terme en français. Comme démontré dans un travail précédent (Sene Mongaba 2012), le terme en lingála contribue également à motiver les élèves dans l'apprentissage de la leçon et à améliorer leur attitude face à leur propre langue.

Comme indiqué plus haut, l'enseignant est souvent amené à réexpliquer sa leçon en lingála lorsque les élèves ne comprennent pas le discours en français. La présentation du document sous forme de dictionnaire devra ainsi permettre à l'enseignant de faire une consultation ciblée, même en cours de leçon, pour trouver le terme équivalent ainsi que l'énoncé de la définition en lingála. Cette approche contribuera ainsi à réduire l'approximation du discours des enseignants. Le dictionnaire leur permettra d'éviter l'usage de mots génériques de la langue générale en lieu et place des termes de spécialité.

Situation 2. Dans son travail de reformulation didactique et linguistique: face à une nouvelle pratique qu'il a pu exprimer en lingála, l'élève ou l'enseignant peut être amené à vouloir transférer cette pratique en français. Dans ce cas, il cherchera un ou plusieurs mots dans le dictionnaire et trouvera son ou ses équivalents en français. En plus, grâce à la définition de ces mots en lingála, il 
pourra aussi se rendre compte si ce terme lingála est adapté au concept qui désigne cette notion ou s'il n'est pas adapté. Dans le dernier cas, grâce aux définitions de différents mots dérivant du même radical, l'utilisateur pourra alors trouver le terme adéquat. Les différents renvois en lingála lui permettront, le cas échéant, de trouver le mot le plus adapté et ainsi l'apprenant pourra fixer durablement ces nouvelles notions apprises. Ceci est illustré par l'exemple sur le radical -sang- (mettre ensemble) que nous exposons plus loin dans ce travail.

Sur base des réalités sociolinguistiques décrites plus haut, nous pouvons affirmer que c'est le lingála facile qui est le plus accessible aux élèves. Par conséquent, les définitions et les exemples dans le dictionnaire seront énoncés dans ce registre lingála facile lf (les termes de spécialités, les nombres, les grandeurs sont en français), même si nous indiquerons entre parenthèses, des termes équivalents dans le registre élaboré lingála ya solo ls. La traduction en français, qui apparaît dans la colonne de droite, contribue à aider les utilisateurs dans leur travail de reformulation, étant donné qu'en classe ils sont obligés d'écrire en français.

\section{Sélections des lemmes et des définitions}

À l'heure actuelle, il n'existe qu'une liste de noms d'éléments chimiques (Mbikay 2001; Sene Mongaba 2009) et deux petits livres de chimie écrits en lingála: Lexique de chimie français-lingála (Mukinayi 2011a) et Shimie, mambi ma ebandela (Mukinayi 2011b). Les publications de Mukinayi constituent une introduction au cours de chimie générale destinée aux étudiants de la première année d'université. Elles ne couvrent pas le programme de chimie de la troisième année secondaire et en plus ils abordent des détails qui vont au-delà de ce qui est prévu pour la troisième année secondaire. Le lingála étant donc une langue très faiblement documentée en matière de chimie, nous avons donc dû, au même moment, tout en rédigeant un livre de chimie pour cette classe, créer aussi des termes de spécialité. Ce manuscrit du livre de chimie ainsi que les deux publications de Mukinayi et la liste des éléments chimiques de Mbikay et complété par Sene Mongaba nous ont servi de corpus. Les définitions en lingála contenues dans ce dictionnaire sont donc des définitions que nous avons produites tout au long de la rédaction de ce livre de chimie. Évidemment, nous avons consulté une bibliographie circonstanciée, constituée des livres de chimie écrits en français et destinés aux écoles congolaises (Mikalukalu 1984, 1986 et 1992, Lufimpadio 1998, Ndjungu Brahimu 2002), ainsi que d'autres livres des écoles secondaires d'autres pays comme la Belgique (Pirson et al. 2009), la Grande Bretagne (Ryan 2006), l'Italie (Tottola et al. 2008), l'Espagne (Del Barrio et al. 2008), la Malaisie (Yasin 2011), le Canada (Kotz et Treichel 2006) et les États-Unis d'Amérique (Moore 2004).

Sur le plan de la terminologie, nous avons d'abord cherché à expliquer, puis à définir le concept en lingála, sans nous soucier de comment cette notion 
allait être désignée en lingála. Une fois la définition produite et le concept contextualisé à l'aide d'exemples, le terme de spécialité en lingála adapté à cette définition du concept découle de l'énoncé. Pour cela, notre connaissance des mécanismes de formation des mots en lingála (la dérivation, la composition, l'adaptation morphologique et phonologique des compositions savantes et l'emprunt) a été d'une grande utilité.

Enfin, nous avons travaillé en lingála en nous basant aussi sur la manière dont les différents locuteurs et auteurs des textes généraux contenus dans le corpus s'y sont pris pour définir les concepts dans cette langue de façon compréhensible. Les textes en français contenus dans les dictionnaires sont donc des traductions des textes lingála.

L'avantage de cette démarche est de constater directement si le terme créé s'insère aisément dans la syntaxe du lingála avec le moins d'ambiguïté possible.

Dans le cadre de ce travail, nous avons utilisé le logiciel Notepad++ pour la constitution du corpus. Le logiciel Unitex a été utilisé pour l'extraction des termes dans le corpus et pour évaluer leur fréquence et leur collocation. Le logiciel TswaneLex a servi pour la création du dictionnaire. Les logiciels Unitex et TswaneLex ont été localisés en lingála. Cela nous a permis de travailler dans un environnement informatique rédigé en lingála et obéissant à la structure linguistique et grammaticale du lingála. Cet aspect de notre travail ne rentre pas dans le cadre de cet article.

\section{Présentation du dictionnaire et structures des articles}

Le dictionnaire est composé de trois parties.

La première partie comporte les explications, en lingála, sur la façon d'utiliser le dictionnaire, les abréviations utilisées, un résumé sur la morphologie du lingála, le marquage des tons et la variété du lingála utilisée. Elle comporte aussi un guide de l'utilisateur sur la manière la plus efficace de rechercher un terme et les explications sur le concept désigné.

La deuxième et la troisième partie constituent le dictionnaire proprement dit. Chaque article apparaît dans un ordre alphabétique général des termes définis. Nous avons adopté l'ordre alphabétique par soucis d'accessibilité directe à l'information. Cette présentation est bénéfique à l'élève qui ne maîtrise pas encore la structure de son cours de chimie. Cette présentation est aussi favorable à l'enseignant qui peut s'y référer pendant qu'il enseigne. Le système de renvoi pallie le risque que l'utilisateur encourt en n'ayant pas une vue globale avec les autres articles en lien avec le concept défini.

La deuxième partie du dictionnaire concerne la direction français-lingála. Dans cette deuxième partie, il y a deux catégories d'articles: les articles du langage de spécialité et les articles du langage général.

Dans la première catégorie, il s'agit d'articles relatifs à des termes chimiques. Ces articles sont constitués des champs suivants: 
(1) Le premier champ est l'entrée elle-même. Le terme chimique est en français. Il correspond à l'une des notions de chimie prévues dans le programme de la troisième année.

(2) Le champ na Lingála correspond au terme chimique équivalent en lingála.

(3) Le champ ezalíníni correspond à la définition chimique de la notion. Cette définition peut aussi être précédée d'une contextualisation dans le langage général, si cela est nécessaire. L'énoncé est fait en lingála avec des termes de spécialité en français.

(4) Le champ ndakisa donne un ou plusieurs exemples de chimie, toujours en lingála, avec des termes de spécialité en français. Il arrive aussi que des exemples de la vie courante soient proposés dans le but de contextualiser le concept et de lui apporter un aspect concret.

(5) Le dernier champ tálá pé correspond aux renvois permettant à l'utilisateur d'approfondir le sujet ou d'avoir une vue plus globale.

Tout l'article est traduit en français, dans le but d'aider l'élève ou l'enseignant à reformuler son discours en français. La page se présente donc sous forme d'un tableau à deux colonnes, où la colonne de gauche concerne le dictionnaire en lingála et la colonne de droite correspond à la traduction française du texte qui est à gauche.

Mélange hétérogène: sangísa ekesáná. Banzóto na shimí tó bacorps chimique, míbalé tó leká, etíámí esíká mókó, kasi, nzóto na nzóto ezalí komónana na ndéngé na yangó. Lisangá wáná ndé babéngaka mélange hétérogène tó sangísa ekesáná.

Ndakisa: Sókí otié pilipíli, matungúlu pé pondú na eboka. Lisangá wáná ezalí mélange hétérogène tó sangísa ekesáná, pó, elóko na elóko ezalí komónana na ndéngé na yangó. Tángo otútí yangó, na súka ekokóma komónana káka néti elóko mókó: pondú batútá. Wáná ekómí mélange homogène.

Tálá pé MÉLANGE, MÉLANGE HOMOGÈNE, SANGÍSA.
Mélange hétérogène: sangísa ekesáná. Deux ou plusieurs corps chimiques sont mis ensemble ou mélangés, mais chaque corps garde son apparence. Cet ensemble est appelé mélange homogène ou sangísa ekesáná.

Exemple: Si l'on met du piment, de l'oignon et du pondú (feuille de manioc) dans un mortier, cet ensemble constitue un mélange hétérogène, puisque l'on voit séparément chaque ingrédient. Si vous pilez le tout, à la fin vous obtenez le pondú pilé (pondú batútá), qui est donc devenu un mélange homogène, puisque le tout ne se présente plus que comme un seul corps.

Voir aussi MÉLANGE, MÉLANGE HOMOGÈNE, SANGÍSA

Mélange homogène: sangísa esangáná.
Elingí koloba, banzóto ezalákí ya
kokesana, esangání, ekómí komónana
néti nzóto káka mókó.

Mélange homogène: sangísa esangáná. néti nzóto káka mókó.

\section{Mélange homogène: sangísa esangáná.} Cela signifie que des corps qui étaient distincts se mélangent et ne paraissent que comme un seul corps. 
Ndakisa: tálá na mélange hétérogène Tálá pé MÉLANGE, MÉLANGE HOMOGÈNE, SANGÍSA
Exemple: voir mélange hétérogène. Voir aussi MÉLANGE, MÉLANGE HOMOGÈNE, SANGÍSA

\section{Noyau: mukókólí. \\ Noyau ezalí motéma ya atome.}

Ndakisa: Mukókólí ya manga ezalí noyau ya manga.

Atome ekabwáná na biténi míbalé: noyau pé baélectron. Na káti ya mukókólí ya atome sóngóló, okokúta baproton, baneutron pé biténi misúsu ya mikélékété. Na libándá ya mukókólí, okokúta baélectron ezalí kotepatepa na bacouche électronique to banzíngánzíngá ekabwáná na milongó.

Ndakisa: Noyau ya atome ya $\mathrm{H}$, idrojeni (hydrogène), ezalí na proton mókó. Oyo ya $\mathrm{Pb}$, mbódi (plomb), ezalí na baproton 82.

Tálá pé ATOME, NOMBRE DE MASSE, NUMÉRO ATOMIQUE, ÉLECTRON, PROTON, NEUTRON.

\section{Soluté: emelami.}

Ezalí corps chimique (nzóto na shimi) óyo ya kantité ya moké emelamaka na óyo ya kantité ya míngi. Óyo ya míngi babéngaka yangó solvant (emela). Lisangá ya emela ná emelami epésaka solution (lisangana).

Ndakisa: Sókí otié sukáli na káti ya mái pó na kosála mái ya lóbo. Sukáli ezalí emelami tó soluté. Mái ezalí emela tó solvant.

Tála pé SOLVANT, SOLUTION, KOSANGANA, KOSANGANISA, EMELA, LISANGANA, KOMELAMA, KOMELAMISA
Noyau: mukókólí.

Le noyau est le cœur de l'atome.

Exemple: Le mukókólí de la mangue est le noyau de la mangue.

L'atome est subdivisé en deux parties, le noyau et les électrons. Au sein du noyau, nous rencontrons des protons, des neutrons et d'autres particules.

En dehors du noyau se trouvent des électrons qui sont dans des couches électroniques tout autour du noyau.

Exemple: Le noyau de l'atome H (hydrogène) a un proton. Celui de l'atome de $\mathrm{Pb}$ (plomb) en a 82.

Voir aussi ATOME, NOMBRE DE MASSE, NUMÉRO ATOMIQUE, ÉLECTRON, PROTON, NEUTRON.

\section{Solvant: emela.}

Na chimie: Ezalí corps chimique (nzóto na shimî) óyo ezalí na kantité ya míngi pé emelaka banzóto na shimí misúsu ya bakantité ya miké (bimelami tó basolutê) pé yangó nyónso ekosangana pó na kosála lisangana tó solution.

Ndakisa: Sókí batié múngwa ná sukáli esíká mókó, ekozala ya kosangisa kasi

\section{Soluté: emelami.}

Un corps chimique généralement en faible quantité qui est dissout dans un autre corps chimique en grande quantité. Ce dernier est appelé solvant. L'ensemble forme la solution.

Exemple: Lorsqu'on dissout du sucre dans l'eau pour faire de l'eau sucrée, le sucre est le soluté tandis que l'eau est du solvant.

Voir aussi SOLVANT, SOLUTION, KOSANGANA, KOSANGANISA, EMELA, LISANGANA, KOMELAMA, KOMELAMISA 
esángáná té. Sókí babakísi mái, babalólí, okomóna esangání. Mái emelí bangó. Mái ezalí emela tó solvant.

Tálá pé SOLVANT, SOLUTÉ, EMELAMI, SOLUTION, KOSANGANISA, KOSANGANA, KOSANGISA hétérogène (juste mis ensemble), mais ils ne se sont pas encore mélangés de façon homogène. Si on ajoute de l'eau, et que l'on agite, ils vont se mélanger de façon homogène. L'eau les a dissous. L'eau est le solvant.

Voir aussi SOLVANT, SOLUTÉ, SOLUTION, KOSANGANISA, KOSANGANA, KOSANGISA

Dans la deuxième catégorie d'articles, il s'agit de termes de langage général en français ayant une occurrence conséquente dans le discours de chimie et qui ont un équivalent lingála. Il s'agit en fait de termes répertoriés et définis dans la partie lingála-français. Les articles sont composés des champs suivants:

(1) Le premier champ est l'entrée elle-même en français;

(2) Le champ na Lingála correspondant à l'équivalent en lingála;

(3) Le champ tála pé correspond aux éventuels renvois.

Dans cette partie, les définitions et les exemples ne figurent pas dans l'article, car ils sont déjà donnés dans l'article équivalent en lingála.

$$
\begin{aligned}
& \text { Mélange: sangísa. } \\
& \text { Tálá pé MÉLANGE HOMOGÈNE, MÉLANGE }
\end{aligned}
$$
HÉTÉROGÈNE, MÉLANGER, SE MÉLANGER
Mélange: sangísa.

Voir aussi MÉLANGE HOMOGĖNE, MÉLANGE HÉTÉROGÈNE, MÉLANGER, SE MÉLANGER

La troisième partie du dictionnaire est consacrée à la direction lingála-français. Le but de cette partie est de permettre à l'utilisateur de prendre conscience des mécanismes linguistiques qu'il utilise inconsciemment en tant que locuteur. Ceci lui permet d'améliorer la compréhension des énoncés définitoires des termes de spécialité figurant dans la deuxième partie. Dans cette troisième partie, il y a aussi deux sortes d'articles: les articles du langage de spécialité et les articles du langage général. Dans le premier cas, l'article est un terme lingála équivalent à un terme de chimie en français. Il s'agit en fait des termes répertoriés et définis dans la partie français-lingála. Dans le deuxième type, l'article peut être un verbe ou un substantif susceptible d'être utilisé lors de la reformulation des leçons de chimie en lingála. Les structures de ces deux types d'articles sont différentes.

Les articles correspondants aux termes de chimie en lingála sont composés de champs suivants:

(1) Le premier champ est l'entrée elle-même en lingála;

(2) Le champ na français correspondant à l'équivalent en français;

(3) Le champ tála pé correspond aux éventuels renvois. 


\begin{tabular}{|l|l|}
\hline $\begin{array}{c}\text { Bobóngolani na shimí: bo-bóng-ol-an-i/ } \\
\text { réaction chimique }\end{array}$ & Bobóngolani na shimí: réaction chimique \\
$\begin{array}{l}\text { Tálá pé KOBÓNGOLA, KOBÓNGOLANA, } \\
\text { KOBÓNGWAMA, LIKOKANI NA SHIMÍ }\end{array}$ & $\begin{array}{l}\text { Voir aussi KOBÓNGOLA, KOBÓNGOLANA, } \\
\text { KOBÓNGWAMA, LIKOKANI NA SHIMÍ }\end{array}$ \\
\hline
\end{tabular}

\begin{tabular}{|c|c|}
\hline $\begin{array}{l}\text { Emela: e-mel-a/solvant. } \\
\text { Tálá pé SOLVANT, SOLUTÉ, EMELAMI, } \\
\text { SOLUTION, KOSANGANISA, KOSANGANA, } \\
\text { KOSANGISA }\end{array}$ & $\begin{array}{l}\text { Emela: solvant. } \\
\text { Voir aussi SOLVANT, SOLUTÉ, EMELAMI, } \\
\text { SOLUTION, KOSANGANISA, KOSANGANA, } \\
\text { KOSANGISA }\end{array}$ \\
\hline
\end{tabular}

Le dictionnaire contient aussi des termes de la langue générale (verbes ou substantifs) qui sont susceptibles d'être utilisés par les enseignants et les élèves dans la reformulation. Les articles relatifs à ces termes comportent des champs supplémentaires. L'énoncé est fait en lingála avec des termes de spécialité en français. Il s'agit des champs suivants:

(1) Le premier champ est l'entrée elle-même (avec éventuellement sa forme plurielle);

(2) Le deuxième champ correspond à la structure morphologique;

(3) Le champ na français, correspondant à l'équivalent en français;

(4) Le champ ezalíníni correspond à la définition (éventuellement dérivationnelle) de l'entrée. Dans ce champ, deux sous-champs sont prévus: na ndéngé ya chimie correspond à la définition chimique du terme et na ndéngé ya batu nyónso qui correspond à une définition dans le langage général;

(5) Le champ ndakisa est réservé aux exemples de chimie et/ou de contextualisation;

(6) Le dernier champ tálá pé correspond aux renvois permettant à l'utilisateur d'approfondir le sujet ou d'avoir une vue plus globale.

Nous illustrons ce type d'article par quelques termes issus du radical -sang-. En chimie, plusieurs concepts relatifs à la notion de mélange, de mise en commun ou de contact interviennent dans la description des processus et des phénomènes. L'enseignant ou l'élève peut être amené à parler d'un de ces concepts. Dans le langage général, l'élève utilisera plus spontanément un verbe du radical -sang-, qui évoque l'idée de mettre ensemble. Les verbes kosangisa (mettre ensemble, mélanger) et kosangana (se mélanger) sont couramment utilisés dans le langage général. $\mathrm{Si}$, par exemple, l'élève veut reformuler la notion de homogénéiser un mélange, il pourra éventuellement utiliser l'un de ces deux verbes. En vérifiant dans le dictionnaire, il verra que les définitions de ces deux verbes ne correspondent pas à la notion qu'il veut exprimer. Le renvoi lui permettra, par exemple, de consulter aussi le terme kosanganisa, qui est un terme moins usité par des locuteurs du lingála, mais qui est plus précis pour désigner le concept d'homogénéisation. Les renvois permettent à l'utilisateur de trouver le terme 
correspondant au concept.

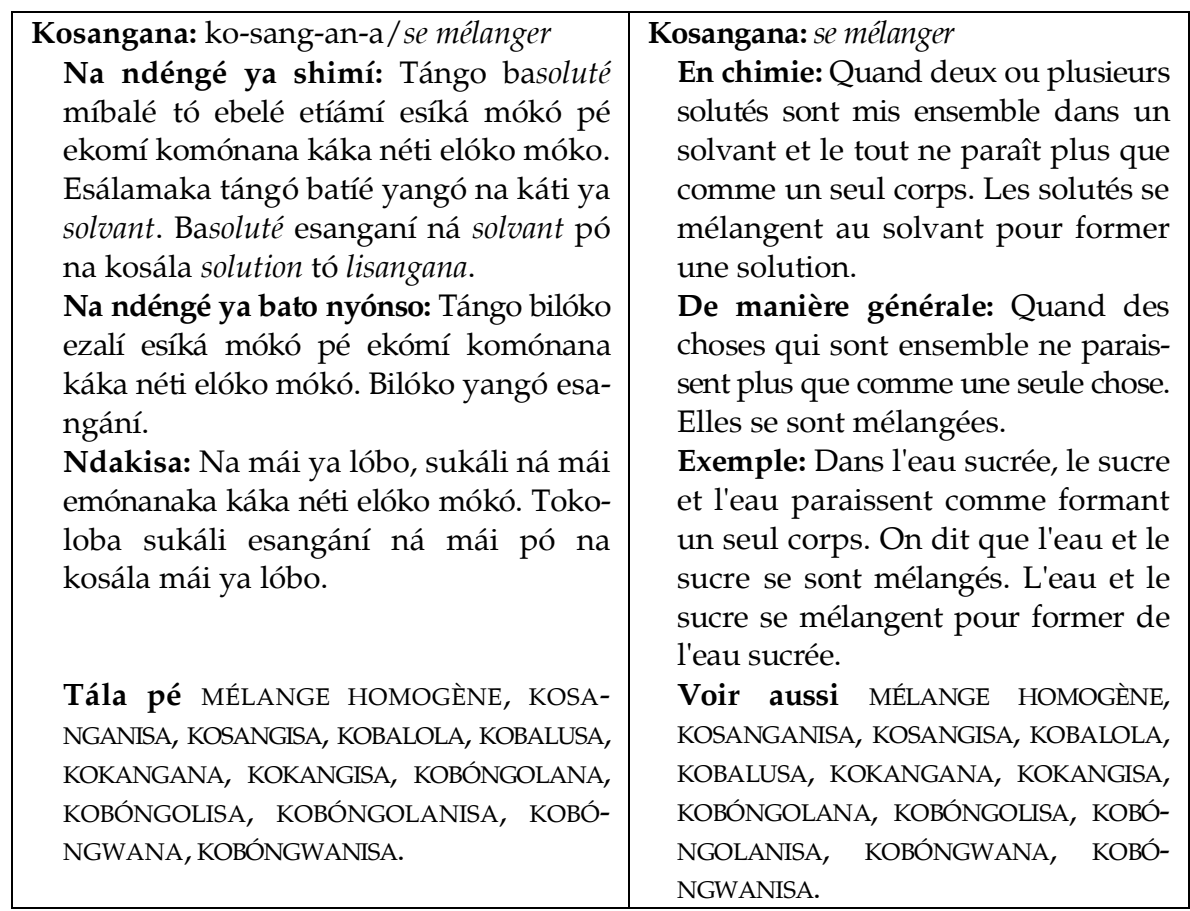

\begin{tabular}{|c|c|}
\hline $\begin{array}{l}\text { osangisa: ko-sang-is-a/mélanger } \\
\text { Na ndéngé ya shimí: Tango otíé bacorps } \\
\text { chimique (banzóto na shimi) míbalé tó } \\
\text { ebelé esíká mókó. Bacorps yangó ekokí } \\
\text { komónana néti elóko mókó (mélange } \\
\text { homogène) tó pé elóko na elóko ekokí } \\
\text { komónana na ndéngé na yangó (mélange } \\
\text { hétérogène). } \\
\text { Na ndéngé ya bato nyónso: Kotía bilóko } \\
\text { ékóma esíká mókó. } \\
\text { Ndakisa: Sókí batíé sukáli na kópo. } \\
\text { Babakísí míliki ya pusyér. Babalúsí ya- } \\
\text { ngó na lútu. Elingí koloba, basangísí ya- } \\
\text { ngó. } \\
\text { Tála pé SANGÍSA, MÉLANGE HÉTÉROGÈNE, } \\
\text { MÉLANGE HOMOGĖNE, KOSANGANA, KOSA- } \\
\text { NGANISA, KOBALOLA, KOBALUSA, KOKA- } \\
\text { NGANA, KOKANGISA, KOBÓNGOLANA, KOBÓ- } \\
\text { NGOLISA, KOBÓNGOLANISA, KOBÓNGWANA, } \\
\text { KOBÓNGWANISA. }\end{array}$ & $\begin{array}{l}\text { En chimie: Quand on met deux ou } \\
\text { plusieurs corps chimiques ensemble. } \\
\text { Ces corps mélangés peuvent paraître } \\
\text { comme un seul corps (mélange homo- } \\
\text { gène) ou chaque corps peut appa- } \\
\text { raître distinctement (mélange hétéro- } \\
\text { gène). } \\
\text { De manière générale: Mettre les } \\
\text { choses ensemble. } \\
\text { Exemple: On met du sucre dans un } \\
\text { gobelet et on y ajoute du lait en } \\
\text { poudre. Ensuite, à l'aide d'une cuil- } \\
\text { lère, on mélange. } \\
\text { Voir aussi SANGÍSA, MÉLANGE HÉTÉ- } \\
\text { ROGĖNE, MÉLANGE HOMOGÈNE, KOSA- } \\
\text { NGANA, KOSANGANISA, KOBALOLA, } \\
\text { KOBALUSA, KOKANGANA, KOKANGISA, } \\
\text { KOBÓNGOLANA, KOBÓNGOLISA, KOBÓ- } \\
\text { NGOLANISA, KOBÓNGWANA, KOBÓ- } \\
\text { NGWANISA. }\end{array}$ \\
\hline
\end{tabular}




\begin{tabular}{|c|c|}
\hline Cosanganisa: ko-sang-an & Kosanganisa: $h o$ \\
\hline $\begin{array}{l}\text { Kosanganisa tó kohomogénéiser ekeséní ná } \\
\text { kosangana. Ekeséní pé ná kosangisa. }\end{array}$ & $\begin{array}{l}\text { Homogénéiser ou kosanganisa diffère de } \\
\text { mélanger (kosangisa) ou se mélanger } \\
\text { (kosangana). }\end{array}$ \\
\hline $\begin{array}{l}\text { Na ndéngé ya shimí: Kosála ke bacorps } \\
\text { chimique (banzóto na shimí), míbalé tó } \\
\text { leká, ésangana yangó na yangó pé ékela } \\
\text { sangísa esangáná tó mélange homogène. }\end{array}$ & $\begin{array}{l}\text { En chimie: Amener deux ou plusieurs } \\
\text { corps chimiques à se mélanger pour } \\
\text { former un mélange homogène. }\end{array}$ \\
\hline $\begin{array}{l}\text { Na ndéngé ya bato nyónso: Kosála ke } \\
\text { bilóko ésangana yangó na yangó tíi éko- } \\
\text { ma néti elóko móko. }\end{array}$ & $\begin{array}{l}\text { En général: Faire que deux ou plu- } \\
\text { sieurs objets se mélangent de façon } \\
\text { à ne paraître que comme un seul } \\
\text { objet. }\end{array}$ \\
\hline $\begin{array}{l}\text { Ndakisa: } 1 \text {. Sókí otíe pilipíli ná múngwa } \\
\text { na eboka. Wáná osangísí pilipíli ná } \\
\text { múngwa (kosangisa). Sikóyo tángu otútí } \\
\text { yangó pé esangání ekómí káka poto- } \\
\text { póto mókó (kosangana). }\end{array}$ & $\begin{array}{l}\text { Exemple: } 1 . \text { Si tu mets du piment et } \\
\text { du sel dans un mortier, tu les as } \\
\text { mis ensemble. Tu as mélangé du } \\
\text { piment et du sel. Osangísí du piment } \\
\text { et du sel (mélanger). }\end{array}$ \\
\hline $\begin{array}{l}\text { Yó moto osálí pilipíli ná múngwa ésa- } \\
\text { ngana. Wáná osangánísí yangó (kosa- } \\
\text { nganisa). }\end{array}$ & $\begin{array}{l}\text { Ensuite, quand tu piles, le tout se } \\
\text { mélange et ne forment plus qu'une } \\
\text { seule pâte. Le piment et du sel se } \\
\text { sont mélangés (se mélanger). } \\
\text { C'est toi qui as fait qu'ils se mélangent, } \\
\text { tu les as homogénéisés, osangánísí } \\
\text { yangó. }\end{array}$ \\
\hline $\begin{array}{l}\text { 2. Sókí osangísí mafúta na súde. Obalólí } \\
\text { yangó tíi ebimísí savón. Okomóna bisíká } \\
\text { mosúsu esálí bambumambuma, bisíká } \\
\text { mosúsu potopóto. Okosanganisa yangó } \\
\text { tíi bisíká nyónso ékóma potopóto ya } \\
\text { ndéngé mókó. }\end{array}$ & $\begin{array}{l}\text { 2. Tu mélanges de l'huile avec de la } \\
\text { soude caustique. Tu malaxes jusqu'à } \\
\text { obtenir du savon. Tu verras que par } \\
\text { endroits il y aura des morceaux durs } \\
\text { et par endroits une pâte. Tu vas homo- } \\
\text { généiser ton produit jusqu'à avoir une } \\
\text { pâte homogène. }\end{array}$ \\
\hline Tálá & $\begin{array}{l}\text { Voir aussi KOSANGANA, KOSANGISA, } \\
\text { ESANGANISI, LISANGÁ, LISANGANI. }\end{array}$ \\
\hline
\end{tabular}

\section{Conclusion}

Cet article a décrit le travail de conception et de rédaction d'un véritable outil pédagogique à l'attention des élèves de la troisième secondaire scientifique. Il s'agit du dictionnaire de chimie lingála-français. Ce dictionnaire respecte deux préalables lexicographiques, qui consistent à caractériser l'utilisateur et à déterminer les objectifs du dictionnaire.

Sur le plan théorique et méthodologique, cette étude a abordé l'approche terminologique dans la confection d'un dictionnaire bilingue langue documentée - langue faiblement documentée. Cette approche consiste à produire du savoir directement dans la langue cible et à le classer ensuite en rapport avec les termes de spécialité extraits de la langue source (documentée). Cette approche 
est différente de l'approche traductologique, qui consiste à extraire les termes, les définitions et les exemples du corpus disponible en langue source et à procéder ensuite à la traduction. Si l'approche terminologique a l'avantage de régler la question de l'usage du terme créé dans la syntaxe de la langue cible, elle nécessite néanmoins que le lexicographe soit aussi expert du domaine ou que le lexicographe travaille avec un expert, qui, lui, va effectivement produire un savoir dans la langue cible. L'approche terminologique est complémentaire à l'approche traductionnelle pour un travail lexicographique global. Ainsi, cette étude a contribué à montrer que la terminologie et la lexicographie répondent, mieux que la linguistique du type saussurien, aux demandes actuelles des locuteurs des langues africaines, afin que ces langues puissent être utilisées comme langues d'enseignement.

Enfin, certains gouvernements (France, Belgique, Afrique du Sud, etc.) disposent de services de terminologie pour la standardisation des propositions (Depecker 2009, Taljard et Nchabeleng 2011). Un organe institutionnel de ce type n'a pas encore été mis en place au Congo, ni dans plusieurs pays d'Afrique. Étant donné que les langues nationales congolaises ne sont pas de langues officielles, le gouvernement congolais n'a pas encore intégré cette dimension dans sa politique linguistique, qui, par ailleurs, n'est pas encore clairement établie et active. La création terminologique reste encore une activité des chercheurs indépendants. Tel est le cadre dans lequel se situent ces travaux de terminologie en lingála. Il serait souhaitable dans l'avenir que des équipes de recherche soient constituées dans ce sens dans les institutions de recherche universitaires $\mathrm{du}$ Congo comme par exemple le centre congolais de terminologie (CECOTEL) qui vient d'être ouvert à l'université de Kinshasa.

\section{Références}

Auger, P. 2001. Essai d'élaboration d'un modèle terminologique/terminographique variationniste. TradTerm 7: 183-224.

Del Barrio, J.I., J. Puente, A. Caamaño et M. Augustench. 2008. Física y Química 1. Madrid: Bachillerato.

Depecker, L. 2009. La terminologie: ouverture d'un champ disciplinaire. Willems, Martine (Éd.). 2009. Pour l'amour des mots: 91-106. Bruxelles: Facultés universitaires Saint-Louis.

Diki-Kidiri, M. 2008. La description de la méthode. Diki-Kidiri, M. et al. (Éds.). 2008. Le vocabulaire scientifique dans les langues africaines: pour une approche culturelle de la terminologie (théorie, méthodologie et applications): 113-142. Paris: Karthala.

Kilosho Kabale, Sim. 2013. Comment gérer le multilinguisme et le plurilinguisme dans les universités des Grands lacs et de l'Afrique centrale. Cas de la RD Congo. Synergies Afrique des Grands Lacs 2: 33-43.

Kotz, J.C. et P.M. Treichel Jr. 2006. Chimie générale. Bruxelles: De Boeck.

Lufimpadio, Nd. 1998. Travaux pratiques de Chimie. Kinshasa: Afrique éditions.

Manduku Sasa, H. 2004. Langues et Communication en milieux estudiantins de Kinshasa. Cas de l'Université de Kinshasa. Penser la Francophonie: 545-551.

Matumele, M., P.A. Ayibite et P. Epanga. 1995. Terminologie grammaticale et pédagogique. Lexique 
Français-Lingála Lingála-Français. Nyembwe, N. (Éd.). 1995. Kinshasa: EUA/CELTA.

Mbikay, D.P. 2001. Molongo mwa kemi. Inédit.

Mikalukalu, K.K.M. 1984. Lexique de chimie. Vol.2. Kinshasa: CRP.

Mikalukalu, K.K.M. 1986. Lexique de chimie. Vol.1. 2 èd. Kinshasa: CRP.

Mikalukalu, K.K.M. 1992. La chimie avec du matériel de fortune. Kinshasa: CRP.

Ministère EPSP RD Congo. 2009. Programme national de chimie. Kinshasa: Édition électronique. http://www.epsp.cd/component/option,com_joomdoc/Itemid,84/gid,52/task,cat_view/

Moore, J.T. 2004. Chemistry Made Simple. New York: Broadway Books.

Mukinayi, J.M. 2011a. Lexique de chimie français-lingála. Kinshasa: Science pour tous.

Mukinayi, J.M. 2011b. Shimie, Mambi ma ebandela. Kinshasa: Science pour tous.

Ndjungu Brahimu, W.K. 2002. Cours de chimie 3ème année. Chimie élémentaire. Kinshasa: CRP.

Nyembwe, N. et T. Koni. 2004. La francophonie en milieux estudiantins de Kinshasa: PratiquePerception-Perspectives. Penser la Francophonie: 471-478.

Nyembwe N. et S. Matabishi. 2012. Le devenir du français en République Démocratique du Congo et question de la norme. Blumenthal, Peter et Stefan Pfänder (Éds.). 2012. Actes du colloque: Convergences, divergences et la question de la norme en Afrique francophone. Le Français en Afrique 27: 109-119.

Pirson, P., H. Bordet, D. Castin, P. Snauwaert et R. Van Elsuwe. 2009. Chimie 3e/4e. Sciences de base et sciences génerales. Bruxelles: De Boeck.

Prinsloo, D., A.R. Chuwa et E. Taljard. The Lexicon of Africa. Webb, Vic et E. Kembo-Sure (Éds.). 2000. African Voices: 220-244. Cape town: Oxford University Press.

Ryan, L. 2006. New Chemistry for You. Revised Edition for all GCSE Examinations. Cheltenham: Nelson Thornes.

Sene Mongaba, B. 2009. Tableau périodique de chimie-Etanda ebandelaka ya bibuki ya kemi. Kinshasa/ Wavre: Mabiki.

Sene Mongaba, B. 2012. The Use of Lingála in the Teaching of Chemistry in DR Congo: A Socioterminological Approach. Marlo, Michael R. et al. 2012. Selected Proceedings of the 42nd Annual Conference on African Linguistics: 308-320. Somerville, MA: Cascadilla Proceedings Project. http://www.lingref.com,document\#2779.

Sene Mongaba, B. 2013a. Lingála na matéya ya nzebi na bitéyelo ya Kinshasa. Búku II. Thèse de doctorat. Gand: Université de Gand.

Sene Mongaba, B. 2013b. Analyse de la diglossie Français-Lingála dans les écoles de Kinshasa. Omer, D. et F. Tupin (Éds.). 2013. Éducations plurilingues: L'aire francophone entre héritages et innovations: 151-166. Rennes: Presses Universitaires de Rennes.

Taljard, E. et M.J. Nchabeleng. 2011. Management and Internal Standardization of Chemistry Terminology: A Northern Sotho Case Study. Lexikos 21: 194-216.

Tottola, F., A. Allegrezza et M. Righetti. 2008. Nuovo corsa di chimica. Milan: Minerva Italica.

Valente, R.S. 2002. La "Lexicologie explicative et combinatoire" dans le traitement des unités lexicales spécialisées. Thèse de doctorat. Montréal: Université de Montréal.

Yasin, Y. 2011. Chemistry SPM 4.5. Petaling Jaya: Sastadi sdn.

Yawidi Mayinzambi, J.P. 2009. Pourquoi mon enfant a échoué. Kinshasa: Mabiki. 